\title{
List of Musical Examples
}

1. Torikagura

3. Odori ji (Hana ni Asobaba)

4. Ō nori

5. Dontappo

6. Ten tsutsu

159

7. Tobisari

160

8. Tataki

161

9. Sandame

162

10. Kata shagir

164

11. Shagiri

166

12. Shidai

167

13. Sanjū

168-169

A. Giday $\bar{u}$

168

B. Giday $\bar{u}$

169

C. Tokiwazu: Shin Yamauba

169

14. Sekkyō kakari

170

15. Özatsuma patterns

171 
